\title{
Manejo clínico y terapéutico de un tumor fibroso pleural con translocación de ALK
}

\author{
Clinical and therapeutic management of a pleural fibrous tumor with ALK translocation
}

Patricia Cruz-Castellanos ${ }^{1 *}$, Laura Gutiérrez-Sainz ${ }^{1}$, Julia Villamayor ${ }^{1}$, María I. Esteban², Alberto Peláez²

y Francisco J. de Castro ${ }^{1}$

${ }^{1}$ Servicio de Oncología Médica; ${ }^{2}$ Servicio de Anatomía Patológica. Hospital Universitario La Paz, Madrid, España

\begin{abstract}
Resumen
Presentamos un caso clínico de un tumor fibroso solitario pleural (TFSP) de comportamiento anómalo por su alta agresividad y mal pronóstico a corto plazo, en el que se identificó una translocación de ALK. EI TFSP es un tumor infrecuente, con una incidencia estimada en torno a 8 casos por 100,000 habitantes y con pocos reportes en la literatura. Lo más habitual es que se trate de una neoplasia de comportamiento benigno, con debut como una masa localizada y cuyo tratamiento de elección es la resección quirúrgica. Todos los datos de la literatura contrastan con nuestra experiencia, que presentamos a continuación.
\end{abstract}

Palabras clave: Tumor fibroso solitario pleural. Translocación ALK. Cáncer.

\begin{abstract}
We present a clinical case of a solitary fibrous pleural tumor (TFSP) with abnormal behavior due to its high aggressiveness and poor short-term prognosis, where an ALK translocation was identified. TFSP is an infrequent tumor, with an estimated incidence of around 8 per 100,000 inhabitants and with few cases reported in the literature. The most common is that it is a neoplasm of benign behavior, with debut as a localized mass and whose The treatment of choice is surgical resection. All the data in the literature contrast with our experience, which we report below.
\end{abstract}

Key words: Solitary fibrous pleural tumor. ALK translocation. Cancer.

Presentamos el caso clínico de un paciente de 45 años, con antecedentes de tabaquismo activo de más de 20 años de evolución. El paciente consultó por síndrome constitucional, asociado a tos seca y disnea de moderados esfuerzos. En la radiografía de tórax se objetivó una consolidación en el lóbulo inferior derecho. El estudio se completó con tomografía computarizada (TC) toracoabdominal y tomografía por emisión de positrones, que confirmó la presencia de una lesión de $17 \mathrm{~cm}$ que ocupaba la totalidad del lóbulo inferior derecho con invasión de la grasa paracardiaca y derrame pleural adyacente (Fig. 1), siendo sugestivo radiológicamente de tumor fibroso solitario pleural (TFSP). Se realizó una biopsia con aguja gruesa en la que se obtuvieron cuatro cilindros que mostraron una tumoración fusiforme moderadamente celular con un estroma esclerótico. Las células que la componían eran de mediano tamaño, con 
citoplasma mal definido y núcleos ovalados vesiculosos, sin intensa anisocariosis ni nucléolo prominente. Se determinó la presencia de una mitosis en cinco campos, y no se observó necrosis en los cilindros estudiados. La inmunohistoquímica mostró expresión de CD99, BCL-2 y CD34, siendo compatibles estos hallazgos con el diagnóstico de TFSP.

El caso se presentó en el comité de tumores para decidir el mejor manejo multidisciplinario de la lesión, dado su gran tamaño, y se decidió resecarla mediante lobectomía. En un primer momento se realizó una exploración mediante toracoscopia derecha bajo anestesia local y sedación profunda, con hallazgo de una gran masa con aspecto intraparenquimatoso. La exploración fue limitada por el gran volumen de la tumoración, que se encontraba adherida a la pleura parietal. En un segundo tiempo se realizó una toracotomía posterolateral derecha con acceso por doble espacio intercostal (quinto y octavo), y a través de ella, una lobectomía inferior derecha con linfadenectomía hiliomediastínica sistemática. La cirugía se realizó sin incidencias importantes y el posoperatorio transcurrió con normalidad.

En la descripción macroscópica de la pieza quirúrgica se destacó la presencia de una gran tumoración de aspecto de sólido, de color blanquecino amarillento con pequeñas zonas quísticas y de necrosis, y focos hemorrágicos (Fig. 1). El tamaño de la lesión era de $17,5 \times 16 \times 9 \mathrm{~cm}$ e infiltraba el parénquima pulmonar adyacente afectando el margen de resección (R1). Desde el punto de vista microscópico, destacaba la gran heterogeneidad tumoral; por un lado, había zonas con intensa celularidad y morfología de célula pequeña, y por otro lado, zonas acelulares formadas por fascículos irregulares de núcleos ovales separados por bandas de colágeno. Asimismo, llamaban la atención las numerosas figuras de mitosis (15 mitosis por 10 campos de gran aumento) y las abundantes zonas de apoptosis y áreas de necrosis tumoral (Fig. 2), lo que contrastaba con la muestra de la biopsia previa. El índice de proliferación celular (Ki 67 ) fue del $80 \%$ en algunas zonas ${ }^{1-3}$. Toda la inmunohistoquímica realizada sobre la pieza tumoral fue negativa: marcadores mesoteliales (calretinina, D2-40, WT-1), epiteliales (AE1/AE3, CK19, CK7, CK5/6, EMA), neuroendocrinos (cromogranina, sinaptofisina, CD56), melánicos (Melan A, S-100) y musculares (actina, desmina). Asimismo, la translocación del sarcoma sinovial también resultó negativa. Dado que en nuestro hospital en ese momento no se disponía de STAT-6, no se pudo determinar este marcador, que habría

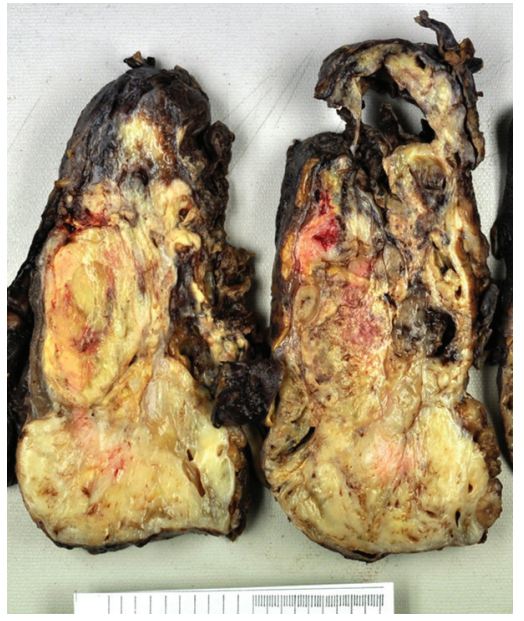

Figura 1. Fotografía macroscópica al corte en la que se muestra la lesión tumoral En el lado izquierdo se observa un corte de la lesión donde se ve su crecimiento difuso y la destrucción del parénquima. En el lado derecho se observa otra zona de la lesión mejor delimitada y respetando el parénquima circundante.

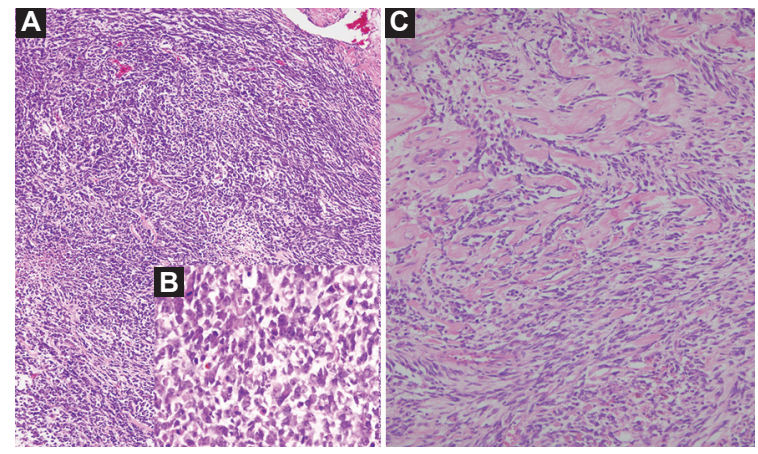

Figura 2. A: fotografía microscópica $(H-E)$ de las zonas hipercelulares. B: numerosas figuras de mitosis. C: áreas más hipocelulares de morfología más convencional de TFSP.

aportado un valor diagnóstico importante dadas sus altas sensibilidad y especificidad para el TFSP. Tras valorar todas las opciones y como diagnóstico de exclusión, se concluyó que la pieza era un TFSP de características malignas.

Al poco tiempo de la intervención, el paciente comenzó con dolor abdominal y elevación de las transaminasas, por lo que se solicitó una TC que mostró lesiones ocupantes de espacio hepáticas de nueva aparición, confirmándose mediante punción con aguja fina su origen metastásico. Debido a lo inusual del caso, en el que nos encontramos ante un TFSP con datos de malignidad y progresión sistémica precoz tras la intervención, para el cual no existe un estándar de tratamiento sistémico, se decidió ampliar con un 

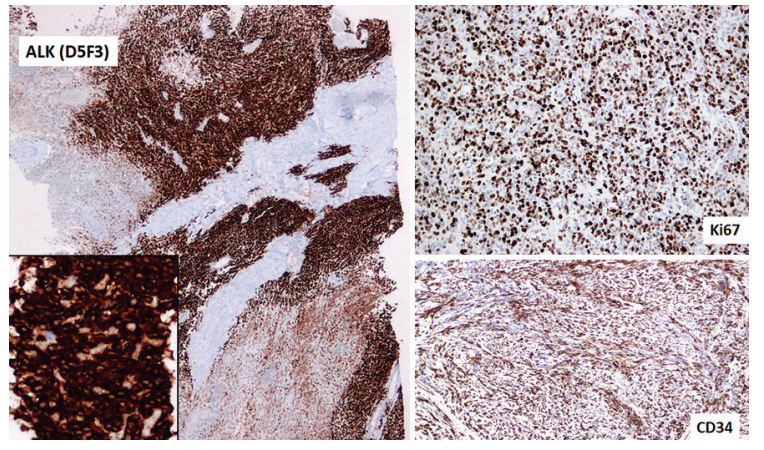

Figura 3. Cortes que muestran la translocación de ALK (con técnica de inmunohistoquímica clon D5F3).

panel mutacional, con el objetivo de encontrar una posible alternativa a la quimioterapia sistémica en una patología infrecuente y actualmente huérfana de tratamiento ${ }^{3}$. Se estudiaron EGFR, ROS 1, c-Kit y ALK, y se encontró expresión de la proteína ALK mediante inmunohistoquímica (Fig. 3). Este hecho se confirmó posteriormente mediante la técnica de hibridación in situ fluorescente, por lo que se planteó el inicio de tratamiento sistémico con crizotinib. Sin embargo, dado el mal estado general del paciente no fue posible, y este falleció poco después a causa de un fallo hepático.

\section{Discusión}

El TFSP es un tumor muy infrecuente que fue descrito por primera vez en $1931^{1}$. Puede presentarse en diferentes localizaciones, incluyendo tejidos blandos y órganos sólidos, pero lo más habitual es que se origine en la cavidad pleural, representando menos del $5 \%$ de todas las neoplasias torácicas. Su etiopatogenia no esta del todo clara, aunque parece probable que tenga un origen mesenquimal. En cuanto a su presentación clínica, lo más habitual es que aparezca entre la quinta y la sexta décadas de la vida, lo cual difiere de nuestro caso, que tuvo una presentación más precoz. Además, no parece haber diferencias por sexo ni claros factores de riesgo identificados ${ }^{2,3}$.

Debido a que se trata de un tumor muy infrecuente, su comportamiento clínico no se conoce por completo. En general se trata de un tumor benigno de crecimiento lento, con síntomas compresivos, como disnea, tos o dolor torácico, como en nuestro paciente. De hecho, por su comportamiento indolente y poco agresivo, suele ser habitual su diagnóstico incidental al realizar una radiografía de tórax por otro motivo ${ }^{4}$.
Por ello, lo más frecuente es que al manifestarse se trate de una enfermedad en estadio localizado, con una masa definida frecuentemente pediculada, de forma que su exéresis puede ser completa. Lo más habitual es que se intervenga mediante videotoracoscopia, con verificación intraoperatoria de márgenes de resección libres. Sin embargo, cuando el tumor se presenta como una gran masa (como en nuestro caso clínico), el abordaje por toracoscopia no es posible y se utilizan otros más radicales, como la toracotomía ampliada. No obstante, la técnica quirúrgica no parece influir en el pronóstico. El único factor que se ha relacionado con un menor riesgo de recaída es la extirpación total de la masa, siendo fundamental dejar unos buenos márgenes de resección.

Las diferentes series comunicadas estiman el riesgo de recaída tras la resección de la lesión en torno al $10-25 \%$, con una supervivencia media a los 10 años del $73-100 \%$,6. El motivo por el cual un TFSP presenta un comportamiento más agresivo de lo esperado, como en nuestro caso, no se conoce. Como factores a analizar es importante determinar en la pieza histológica si existen criterios de alta agresividad: presencia de necrosis, pleomorfismo nuclear, infiltración de tejidos adyacentes (pulmón, partes blandas de la pared torácica), áreas de desdiferenciación y elevado índice mitótico (más de tres mitosis en 10 campos de gran aumento) ${ }^{7}$. Estos datos, junto con la presencia de cirugía incompleta, enfermedad metastásica al debut y tumor $>10 \mathrm{~cm}$ máximo, orientan hacia la presencia de un tumor más agresivo y de peor pronóstico. Se ha publicado un estudio retrospectivo que plantea la posibilidad de una escala con valor predictivo en la recurrencia de este tumor. Dicha escala se ha validado en una muestra de 113 pacientes, en los que se evaluaron el origen pleural o no de la lesión, la morfología, el tamaño, la presencia de necrosis o hemorragia, y el número de mitosis, siendo clasificados los pacientes en bajo o alto riesgo de recurrencia. El tamaño $>10 \mathrm{~cm}$, la hipercelularidad, la necrosis o la hemorragia, y cuatro mitosis por campo se relacionaron con un aumento de la recidiva a largo plazo ${ }^{8}$. Además, este último se identificó como factor independiente de recidiva a largo plazo. Según esta escala, nuestro paciente presentaba un alto riesgo de recaída, lo que se tradujo en una evolución tórpida.

Respecto al tratamiento sistémico del TFSP existen pocos datos, debido a su baja frecuencia y a su comportamiento mayoritariamente benigno. Cuando nuestro paciente progresó de forma precoz tras la 
cirugía, nos planteamos cuál podría ser la mejor opción terapéutica. En cuanto a la posibilidad de quimioterapia, tan solo existen datos de series retrospectivas en las que se ha evaluado el posible papel de las antraciclinas, la ifosfamida, los platinos y los taxanos. Todos estos estudios muestran unas tasas de respuesta pobres, con una supervivencia libre de progresión de unos 4 meses y con una toxicidad importante ${ }^{9-11}$. También se barajó la posibilidad de iniciar tratamiento con inhibidores de la tirosina cinasa, por presentar mejor perfil de toxicidad, con medianas de supervivencia similares a las de los tratamientos citotóxicos ${ }^{12,13}$.

Sin embargo, valorando las escasas opciones terapéuticas disponibles, y dado que existía la posibilidad en nuestro centro de realizar un panel mutacional con el objetivo de encontrar una diana terapéutica en el marco de la medicina personalizada, optamos por esta última opción y encontramos una translocación de ALK, y con ello la posibilidad de un tratamiento eficaz. Como es conocido, esta alteración se usa como diana terapéutica en diferentes tipos tumorales, y existen diferentes fármacos altamente eficaces que han cambiado el pronóstico y la evolución de estas neoplasias. Lo más frecuente es que se encuentre asociada al cáncer de pulmón no microcítico, pero en la literatura se ha descrito la presencia de dicha translocación en ciertos tumores raros, como el histiocitoma fibroso epitelioide y algunos sarcomas de partes blandas poco frecuentes ${ }^{14}$. Cuando se identifica esta translocación se debe iniciar el tratamiento dirigido con inhibidores específicos, como crizotinib, ceritinib o alectinib, obteniendo una rápida respuesta y medianas de supervivencia que pueden alcanzar los 20 meses $^{15}$. En nuestro caso clínico, a pesar de identificar esta diana no se pudo iniciar el tratamiento idóneo debido al rápido deterioro del paciente y su fallecimiento poco después.

Este hecho hace que reflexionemos acerca de si, en la era de la medicina personalizada, tumores raros como el TFSP, de los que se desconoce su evolución clínica y se carece de tratamientos eficaces, no se deberían encuadrar dentro de las enfermedades raras y con necesidad de una buena tipificación mediante técnicas de biología molecular, con el objetivo de un tratamiento personalizado.

\section{Responsabilidades éticas}

Protección de personas y animales. Los autores declaran que para esta investigación no se han realizado experimentos en seres humanos ni en animales.

Confidencialidad de los datos. Los autores declaran que han seguido los protocolos de su centro de trabajo sobre la publicación de datos de pacientes.

Derecho a la privacidad y consentimiento informado. Los autores han obtenido el consentimiento informado de los pacientes y/o sujetos referidos en el artículo. Este documento obra en poder del autor de correspondencia.

\section{Conflicto de intereses}

Los autores declaran no tener conflicto de intereses.

\section{Bibliografía}

1. Klemperer P, Rabin CB. Primary neoplasms of the pleura: a report of five cases. Arch Pathol. 1931;11:385

2. WHO, Travis WD, Tazelaar H, Churg A, Pugatch R, Aubry MC, et al. Pathology and genetics. Tumors of lung, pleura, thymus and heart. WHO editorial. Lyon, Francia, 2004. Disponible en: https://patologi.com/ who\%20lunge.pdf

3. Briselli M, Mark EJ, Dickerson GR. Solitary fibrous tumors of the pleura: eight new cases and review of 360 cases in the literature. Cancer. 1981;47:2678-89.

4. Chick JF, Chauhan NR, Madan R. Solitary fibrous tumors of the thorax: nomenclature, epidemiology, radiologic and pathologic findings, differential diagnoses, and management. AJR Am J Roentgenol. 2013;200:W238.

5. Cardillo G, Lococo F, Carleo F, Martelli M. Solitary fibrous tumors of the pleura. Curr Opin Pulm Med. 2012;18:339.

6. Sung SH, Chang JW, Kim J, Lee KS, Han J, Park SI. Solitary fibrous tumors of the pleura: surgical outcome and clinical course. Ann Thorac Surg. 2005;79:303-7

7. Demicco EG, Park MS, Araujo DM, Fox PS, Bassett RL, Pollock RE, et al. Solitary fibrous tumor: a clinicopathological study of 110 cases and proposed risk assessment model. Mod Pathol. 2012;25:1298-306.

8. Tapias LF, Mercier O, Ghigna MR, Lahon B, Lee H, Mathisen DJ, et al. Validation of a scoring system to predict recurrence of resected solitary fibrous tumors of the pleura. Chest. 2015:147:216-23.

9. Constantinidou A, Jones RL, Olmos D, Thway K, Fisher C, Al-Muderis O, et al. Conventional anthracycline-based chemotherapy has limited efficacy in solitary fibrous tumour. Acta Oncol. 2012;51:550-4

10. Stacchiotti S, Libertini M, Negri T, Palassini E, Gronchi A, Fatigoni S, et al. Response to chemotherapy of solitary fibrous tumour: a retrospective study. Eur J Cancer. 2013;49:2376-83.

11. Park MS, Ravi V, Conley A, Patel SR, Trent JC, Lev DC, et al. The role of chemotherapy in advanced solitary fibrous tumors: a retrospective analysis. Clin Sarcoma Res. 2013;3:7.

12. Stacchiotti S, Negri T, Libertini M, Palassini E, Marrari A, De Troia B, et al. Sunitinib malate in solitary fibrous tumor (SFT). Ann Oncol. 2012;23:3171-9

13. Martín-Broto J, Stacchiotti S, López-Pousa A, Redondo A, Bernabeu D, de Álava $\mathrm{E}$, et al. Pazopanib for treatment of advanced malignant and dedifferentiated solitary fibrous tumour: a multicentre, single-arm, phase 2 trial. Lancet Oncol. 2019;20:134-44.

14. Doyle LA, Mariño-Enríquez A, Fletcher DM, Hornick JL. ALK rearrangement and overexpression in epithelioid fibrous histiocytoma. Mod Pathol. 2015;28:904-12

15. Kwak EL, Bang YJ, Camidge DR, Shaw AT, Solomon B, Maki RG, et al. Anaplastic lymphoma kinase inhibition in non-small-cell lung cancer. N Engl J Med. 2010;363:1693-703. 